\title{
Video Article \\ DiOLISTIC Labeling of Neurons from Rodent and Non-human Primate Brain Slices
}

\author{
Gail K. Seabold ${ }^{1}$, James B. Daunais ${ }^{2}$, Andrew Rau ${ }^{3}$, Kathleen A. Grant ${ }^{3}$, Veronica A. Alvarez ${ }^{1}$ \\ ${ }^{1}$ Section on Neuronal Structure, Laboratory for Integrative Neuroscience, NIAAA, NIH \\ ${ }^{2}$ Department Physiology and Pharmacology, Wake Forest University Health Sciences \\ ${ }^{3}$ Oregon National Primate Research Center, Division of Neuroscience, Oregon Health and Science University
}

Correspondence to: Veronica A. Alvarez at alvarezva@mail.nih.gov

URL: https://www.jove.com/video/2081

DOI: doi:10.3791/2081

Keywords: JoVE Neuroscience, Issue 41, gene gun, dendritic spine, dendrite branching, neuronal morphology, dil, mouse brain, cynomogus monkey brain, fluorescent labeling

Date Published: 7/6/2010

Citation: Seabold, G.K., Daunais, J.B., Rau, A., Grant, K.A., Alvarez, V.A. DiOLISTIC Labeling of Neurons from Rodent and Non-human Primate Brain Slices. J. Vis. Exp. (41), e2081, doi:10.3791/2081 (2010).

\section{Abstract}

DiOLISTIC staining uses the gene gun to introduce fluorescent dyes, such as Dil, into neurons of brain slices (Gan et al., 2009; O'Brien and Lummis, 2007; Gan et al., 2000). Here we provide a detailed description of each step required together with exemplary images of good and bad outcomes that will help when setting up the technique. In our experience, a few steps proved critical for the successful application of DiOLISTICS. These considerations include the quality of the Dil-coated bullets, the extent of fixative exposure, and the concentration of detergent used in the incubation solutions. Tips and solutions for common problems are provided.

This is a versatile labeling technique that can be applied to multiple animal species at a wide range of ages. Unlike other fluorescent labeling techniques that are limited to preparations from young animals or restricted to mice because they rely on the expression of a fluorescent transgene, DiOLISTIC labeling can be applied to animals of all ages, species and genotypes and it can be used in combination with immunostaining to identify a specific subpopulation of cells. Here we demonstrate the use of DiOLISTICS to label neurons in brain slices from adult mice and adult non-human primates with the purpose of quantifying dendrite branching and dendritic spine morphology.

\section{Video Link}

The video component of this article can be found at https://www.jove.com/video/2081/

\section{Protocol}

1. Preparing Dil/Tungsten Bead Bullets: Dil (1-1'-Dioctadecyl-3,3,3',3'- tetramethylindocarbocyanine perchlorate) bullets should be prepared in advance of cutting brain slices. The procedure takes approximately 2-4 hours depending on how many bullets are prepared at once.

1. If starting from a new batch of tungsten beads, prepare aliquots of $300 \mathrm{mg}$ each. Aliquots are prepared by suspending 6 grams of tungsten in $2 \mathrm{ml}$ of methylene chloride. Then, pipette $100 \mu \mathrm{l}$ of bead solution into microfuge tubes to produce aliquots of $300 \mathrm{mg}$ tungsten beads. Store aliquots at room temperature. (Note: methylene chloride evaporates extremely quickly. Alcohol can also be used minimize evaporation).

2. When starting from aliquots of beads, resuspend each aliquot ( $300 \mathrm{mg}$ tungsten beads) in $300 \mu \mathrm{l}$ of methylene chloride and cover quickly to prevent evaporation. This quantity is enough for 3 batches of bullets.

3. Prepare dye solution by weighing $13.5 \mathrm{mg}$ of lipophilic dye Dil and adding $450 \mu \mathrm{l}$ of methylene chloride (final concentration $=3 \mathrm{mg} / 100 \mu \mathrm{l}$ ).

4. Coat the beads with dye. Place a glass slide on a piece of wax-coated weigh paper and pipette $100 \mu \mathrm{l}$ of beads onto the slide. Add $100 \mu \mathrm{l}$ of Dil solution, mix thoroughly using pipette tip, and air dry until beads turn light grey.

5. Use a razor blade to scrape beads off the glass slide and dice the beads into a fine powder. (Note: use a new blade if doing more than one color of bullets so as to not contaminate dyes).

6. Scrape beads onto the weigh paper and funnel beads into a $15 \mathrm{ml}$ conical tube. Add $3 \mathrm{ml}$ of water and sonicate in water bath for $10-30$ min at room temperature. (Note: dicing of the powder and sonication are applied to minimize the formation of clumps of coated beads that will disrupt the sparse labeling of individual neurons).

2. Preparing polyvinylpyrrolidone (PVP) solution and tubing: To improve bead attachment to the bullet tubing (TEZFEL tubing), coat it with polyvinylpyrrolidone (PVP) solution.

1. Prepare $10 \mathrm{ml}$ of PVP solution in water at a concentration of $10 \mathrm{mg} \mathrm{PVP} / \mathrm{ml}$.

2. Use a $12 \mathrm{ml}$ syringe with tubing adaptor (flexible tubing with slightly larger diameter) to pass the PVP solution through the bullet tubing and then expel it out the other end. Reuse solution to coat more bullet tubing if several batches are prepared simultaneously. Discard PVP solution after use. 
3. To add the beads to the bullet tubing, vortex the beads to produce a homogenous solution and use the syringe to pull the bead solution through the tubing so that beads are evenly distributed throughout the tubing. ((Note:) try to minimize the number of air bubbles in the tubing).

4. Feed the tubing through the prep station and allow the beads to settle. Use the syringe to gently remove the water so that only the beads remain.

5. Spin the tubing in the prep station and dry with nitrogen gas until water droplets are no longer visible. This step will take approximately $10-20$ $\min$.

6. Cut bullets with tubing cutter into $13 \mathrm{~mm}$ lengths and place in scintillation vials containing Drierite or some kind of anhydrous calcium sulfate Wrap vials in foil to protect from light.

3. Dil Staining: This labeling technique can be used to stain neurons in brain tissue from diverse species; in this particular case, from mouse (3-6 month old) and non-human primates (9-15 years old).

It is preferable that slices are prepared from fixed brain tissue obtained by transcardiac perfusion of fixative solution because tissue preservation is expected to be best under this condition. However, fixation by perfusion is not always feasible (as was the case with the monkey tissue) and DiOLISTIC labeling can still be successfully applied under different procedures for tissue preparation. Here we describe three different procedures for tissue preparation:

a. Fix brain by transcardiac perfusion $\rightarrow$ remove brain $\rightarrow$ postfix for 10 min $\rightarrow$ cut slice $\rightarrow$ stain

b. Remove brain $\rightarrow$ cut block of tissue $\rightarrow$ fix block $\rightarrow$ wash in PBS $\rightarrow$ cut slice $\rightarrow$ stain

c. Remove brain $\rightarrow$ cut slices $\rightarrow$ fix $\rightarrow$ wash $\rightarrow$ stain

In the present study, the monkey brains were obtained from subjects that were transcardially perfused with ice cold artificial cerebral spinal fluid (ACSF) prior to brain harvest and a block (4 mm thick) of tissue containing the caudate and putamen was fixed for $60 \mathrm{~min}$ at room temperature. For all procedures, fixative solution contains $4 \%$ paraformaldehyde and $4 \%$ sucrose in PBS (prepared fresh or used within 15 days). It is important to note that fixation times are critical for successful staining. Overfixation can affect staining by disrupting the integrity of the plasma membrane and causing dye to leak out of the cell (see more information under results).

1. For procedure $a$ and $b$, slices $(200 \mu \mathrm{m})$ are cut from fixed brain or tissue block and slices placed in 24 -well plate with PBS. For procedure

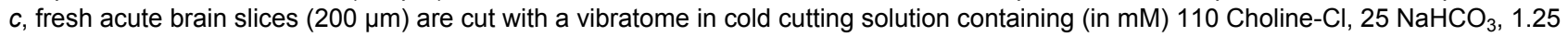
$\mathrm{NaH}_{2} \mathrm{PO}_{4}, 2.5 \mathrm{KCl}, 0.5 \mathrm{CaCl}_{2}, 7 \mathrm{MgCl}_{2}, 25$ glucose, 11.6 ascorbic acid, 3.1 pyruvic acid (osmolarity = 310 mOsmols) and immediately after slices are placed in 24-well plates and fixed for 30 min with fixative solution at room temperature.

2. Wash the slices with PBS, 3 times.

3. Before shooting slices, remove the PBS from the wells and, using a paintbrush, place the slice in the center of the well.

4. Shoot Dil bullets through 3.0 $\mu \mathrm{m}$ filter paper using a Helios Gene Gun system at 120-180 psi helium gas pressure by placing the gun at a distance of $1.5 \mathrm{~cm}$ between the sample and the end of the barrel.

(Important note: Only neurons within $50 \mathrm{~mm}$ of the slice surface will be labeled by this method so it is important to keep the same side of the slice facing up during the washes and for mounting. In this way, you will make sure that the labeled neurons will be within focal distance when imaging with a confocal microscope).

5. Wash slices with PBS 3 times and store them in PBS for several hours to allow the Dil to spread along the dendrites.

6. Mount slices onto a glass slide with ProLong Gold Antifade and cover with an $18 X 18 \mathrm{~mm}$ No.1 coverslip. (Note: for procedure $c$, it is best to mount slices on the same day as the integrity of the slices is not well maintained overnight).

7. Seal with clear nail polish after mounting media has dried.

8. Alternatively, the slices can be stained with antibodies before mounting as described below. (Note: Slides can be used at least 6 months to a year if stored in the dark at $4^{\circ} \mathrm{C}$. Dil is light sensitive and long-term exposure to light will cause the stain to fade).

4. Antibody Staining: Immunostaining should be performed after step 3.4 and before mounting.

1. Permeabilization: Incubate slices in $\mathbf{0 . 0 1} \%$ Triton $\mathrm{X}-100$ in PBS solution for $15 \mathrm{~min}$ at room temperature $(300-500 \mu \mathrm{l}$ per well). NOTE: Do not use higher concentrations of detergent as this will dissolve Dil and significantly reduce the fluorescent staining. Try to minimize the time in detergent as extensive incubations will also reduce the Dil labeling. If extended incubations are necessary, keep slices in PBS rather than in blocking solution.

2. Blocking: Incubate slices in blocking solution containing $10 \%$ goat serum, $0.01 \%$ Triton $X-100$ in PBS for 30 min at room temperature

3. Primary antibody: Add antibody at the desired dilution in blocking solution (e.g. rabbit anti-GFP antibody at 1:1000 dilution). Add 300-500 $\mu \mathrm{l}$ per well and incubate for 1-2 hrs. (Note: if overnight incubations are necessary, remove detergent from blocking solution).

4. Wash slices with PBS for 5 - 15 min, 3 times.

5. Secondary antibody: Incubate with secondary antibody for $30 \mathrm{~min}$ at room temperature. Prepare secondary antibody solution at desired dilution in blocking solution (e.g. Alexa-Fluor 488 conjugated antibody at 1:1000 dilution).

6. Wash slices with PBS for $5-15$ min, 4 times.

7. Mount slices on slides with ProLong Gold Antifade and cover with an $18 \times 18 \mathrm{~mm}$ No.1 coverslip. Seal with nail polish after mounting media has dried.

\section{Representative Results:}

1. Checking for correct labeling: Section can be visualized under a fluorescent dissecting scope equipped with a mercury lamp and red fluorescent filter cube to confirm successful DiOLISTIC labeling. Figure 1 shows exemplary images of nicely labeled brain sections obtained at low magnification. Two important characteristics to look for are a sparse labeling pattern (Figure $1 \mathrm{~A}$ ) and the ability to identify individual cellular components (Figure 1B-C).

2. Troubleshooting DiOLISTIC labeling: Figure 2 shows examples of sections in which the DiOLISTIC labeling worked incorrectly. In our experience, there are three main causes for inefficient labeling: 
- Labeling is too sparse or dense: Very few cells are labeled per section in one case or too many cells are labeled preventing the isolation and study of single cellular elements. Solution: adjust the concentration of coated beads in the final solution used for coating the TEFZEL bullet tubing (section 1.6). Decrease or increase the volume of water ( $3 \mathrm{ml}$ initial value) used to dissolve the beads in order to correct for too sparse or too dense labeling, respectively. In addition, low efficiency of labeling might be caused by less than optimal gas pressure when shooting the coated beads onto the sections. This possibility can be ruled out by making sure that the TEFZEL bullet tubing has released most of the coated beads and it appears clear after shooting. Otherwise, gas pressure for shooting should be increased.

- Bad bullets: large clumps or clusters of dye-coated tungsten beads are formed during the bullet preparation. Sections will resemble examples in Figure 2A-B. Solution: make new bullets paying particular attention to section 1.5 and/or extend sonication time in section 1.6.

- Over-fixation: tissue is kept in paraformaldehyde solution for longer than the optimally required time for fixing (30 minutes for $200-300$ $\mu \mathrm{m}$ sections, 1 hour for $4 \mathrm{~mm}$ sections). This compromises the integrity of the plasma membrane and produces labeled sections that look like those shown in Figure 2C-D in which labeling seems out of focus due to dispersion of dye out of the cells. Solution: reduce fixation time.

3. Confocal imaging: Image stacks are acquired using a confocal microscope (Zeiss LSM 510 META) with a 20x objective for the whole cell and $63 x$ water immersion objective for dendrite segments. Dil was excited using a DPS $561 \mathrm{~nm}$ laser line and green fluorescence from the secondary antibody was excited using a $488 \mathrm{~nm}$ laser line. The optical sectioning of the labeled sample achieved when using confocal microscopy further allows for isolation of single labeled cells in the sample. Figure 3A shows a striatal medium spiny neuron from mouse brain and its complex dendrite branch pattern. High magnification images of dendrite branches of these neurons from rodent (Figure 3B) and non-human primate (Figure 3C) demonstrate the degree of staining in dendrites and dendritic spines using this technique in both species. Dendrites and their protrusions (spines) appear well defined, even when considering the long, thin spine heads so abundant in medium spiny neurons.

When combining DiOLISTICS with immunostaining, confocal imaging is also helpful in unequivocally localizing the stain to the same focal plane and the same cell. Figure 4 shows an example of colocalization of Dil labeling and immunostaining for GFP in a single cell. The image is one stack $(0.7 \mu \mathrm{m} z$-section) obtained from a striatal slice from a transgenic mouse expressing the fluorescent protein GFP under the D1 dopamine receptor promoter.
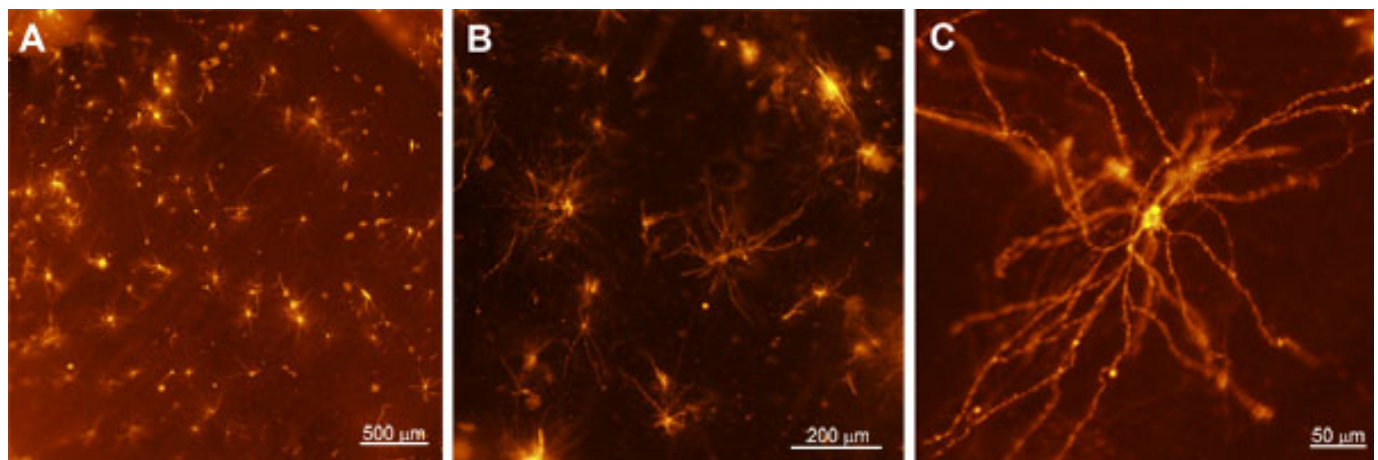

Figure 1. Dil labeling of neurons in brain slices from non-human primates. (A) Low magnification image of a stained brain slice containing the caudate nucleus from a cynomolgus monkey that shows sparse labeling of neurons with Dil. (B-C) Isolated medium spiny neurons can be easily identified using a fluorescent dissecting scope. 

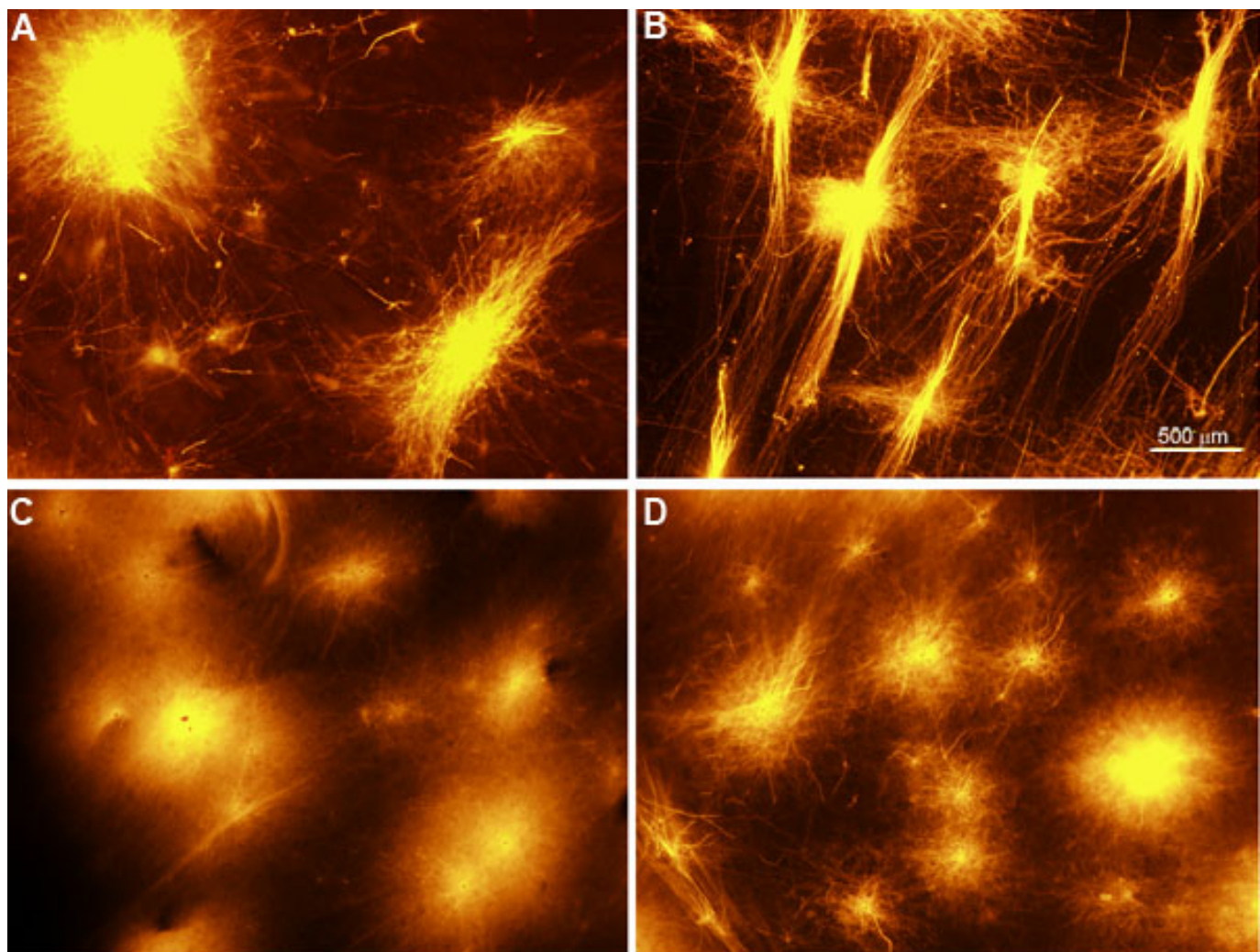

Figure 2. Troubleshooting DiOLISTIC labeling. (A-B) Stained slices from dorsal striatum of monkey (A) and mouse (B) showing large clumps of dye-coated beads and as a consequence, no individual cellular elements can be distinguished. (C-D) Images that exemplify the result of applying DiOLISTIC labeling to sections that were kept in fixative solution for prolonged periods of time or where tissue preservation failed in monkey (C) and mouse (D) tissue.
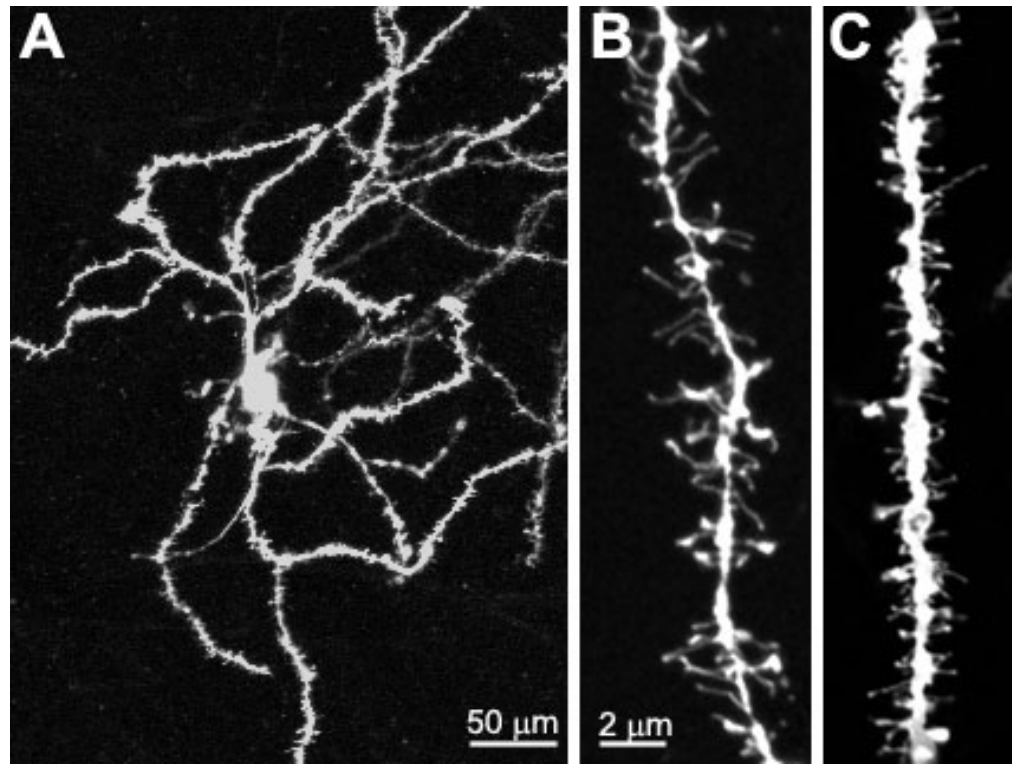

Figure 3. Confocal image of striatal medium spiny neuron stained with Dil. (A), Image stack of a whole cell allows for morphological analysis of the dendritic branches. (B-C) Dendrite segments from mouse (B) and monkey (C) medium spiny neurons show clear labeling of dendrite and spines. 

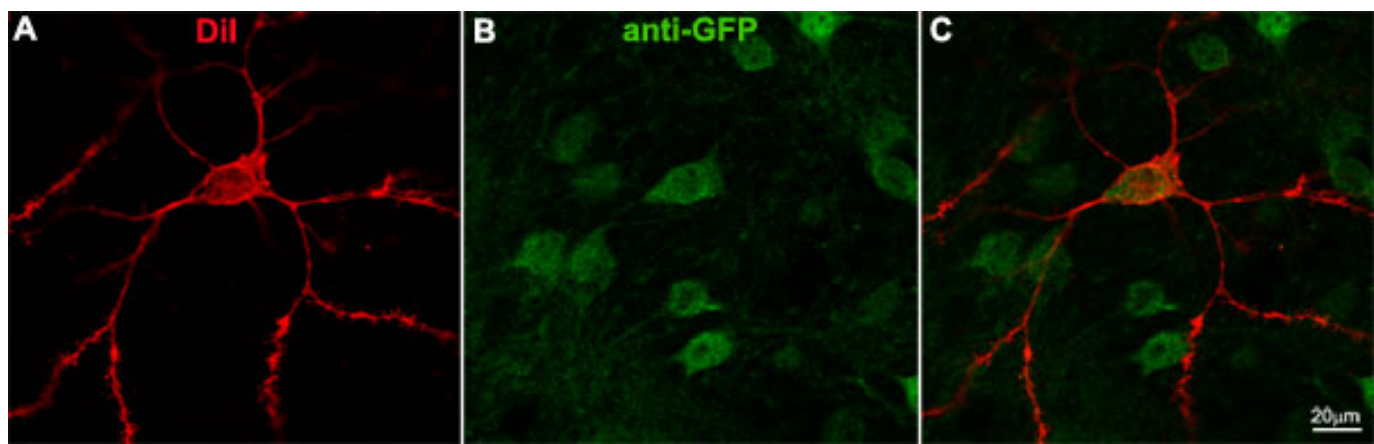

Figure 4. Combining DiOLISTIC labeling with immunostaining. (A) Dil labeled medium spiny neuron from striatum of BAC transgenic mouse expressing GFP under the D1 dopamine receptor promoter. (B) Immunostaining using anti-GFP antibody as described by methods adapted from Lee et al. (2006). Same field as A. (C) Composite image of red and green fluorescence identifies Dil-labeled neuron as a GFP-positive neuron.

Discussion

DiOLISTIC labeling is one of the most versatile techniques available for fluorescently labeling cells because it can be applied to tissue sections from diverse species, to a wide range of ages, and to tissue obtained fresh or from fixative-perfused animals (see also Gan et al., 2009). The process is relatively fast as it takes 1-2 days and it can be combined with other more classical labeling approaches such as immunostaining (Lee et al., 2006). Special attention should be paid to avoid over fixing and the use of high detergent concentrations in incubation solutions because these will comprise the integrity of the lipophilic membrane and cause the dye to leak out of the cells. Antibody penetration can be facilitated by low concentrations of Triton X-100 (Lee et al., 2006), as well as digitonin or saponin in the incubation solution (Matsubayashi et al., 2008). Some modifications that can be applied to this technique include the use of bullets with multiple dyes as described by Gan et al. (2000) or changing the length and type of antibody exposure (Neely et al., 2009).

Traditionally, Dil has been used to trace neuronal projections in the brain. DiOLISTIC labeling expands its application by describing a method useful to examine cell morphology. Neuronal morphology is of great interest because of the large diversity found in the brain and the speculation that cell shape might reflect on the function multiplicity of neuronal populations in the nervous system. One example of this is the fact that many neurons in the mammalian nervous system display small protrusion called dendritic spines that are the site of glutamatergic synapses. In this way, the density of glutamatergic synapses onto a cell can be correlated to the density of dendritic spines, which can be measured using the labeling technique described herein. Furthermore, other morphological parameters such as dendrite total length, branching pattern, dendritic spine shape and density can be quantified and studied.

The use of fluorescent labeling for studying neuronal morphology has many advantages when compared to more traditional techniques that rely on bright field microscopy (e.g. Golgi staining) because it allows for higher resolution confocal imaging. Another advantage of using Dil as a fluorophore in experiments aimed at measuring dendritic spine density and morphology is its lipophilic properties. Dil partitions into the plasma membrane and provides a well defined outline of the neuronal processes and dendritic protrusions. Given the small volume of most dendritic spines (less than 1 femtoliter), membrane staining is more efficient and allows for better visualization of small, thin protrusions than cytoplasmic staining.

\section{Disclosures}

All animal procedures were performed following guidance from the Animal Care and Use Committee at NIAAA and the Oregon National Primate Research Center. The authors have nothing to disclose.

\section{Acknowledgements}

We would like to acknowledge Michael Feyder and Terrell Holloway for their assistance during the initial set up of the technique and Dr. Fumi Ono s laboratory for the access to their confocal microscope. This research was funded by the National Institute of Health through the intramural program of NIAAA and NINDs.

\section{References}

1. Gan, W.B., Grutzendler, J., Wong, W.T., Wong, R.O., Lichtman, J.W., Multicolor "DiOLISTIC" labeling of the nervous system using lipophilic dye combinations. Neuron 27, 219-225 (2000).

2. Gan, W.B., Grutzendler, J., Wong, R.O., and Lichtman, J.W. (2009). Ballistic delivery of dyes for structural and functional studies of the nervous system. CSH Protoc pdb prot5202 (2009).

3. Grutzendler, J., Tsai, J., \& Gan, W.B. Rapid labeling of neuronal populations by ballistic delivery of fluorescent dyes. Methods $30,79-85$ (2003).

4. Lee, K.W., Kim, Y., Kim, A.M., Helmin, K., Nairn, A.C., Greengard, P. Cocaine-induced dendritic spine formation in D1 and D2 dopamine receptor-containing medium spiny neurons in nucleus accumbens. Proc. Natl. Acad. Sci. U S A 103, 3399-3404 (2006).

5. Matsubayashi, Y., Iwai, L., Kawasaki, H. Fluorescent double-labeling with carbocyanine neuronal tracing and immunohistochemistry using a cholesterol-specific detergent digitonin. J Neurosci Methods. 174, 71-81 (2008). 
6. Neely, M.D., Stanwood, G.D., Deutch, A.Y. Combination of diOlistic labeling with retrograde tract tracing and immunohistochemistry. J Neurosci Methods. 184, 332-336 (2009).

7. O'Brien, J.A., Lummis, S.C. Diolistics: incorporating fluorescent dyes into biological samples using a gene gun. Trends Biotechnol. 25, 530-534 (2007).

8. Shen, H.W., Toda, S., Moussawi, K., Bouknight, A., Zahm, D.S., Kalivas, P.W. Altered dendritic spine plasticity in cocaine- withdrawn rats. J. Neurosci. 29, 2876-2884 (2009). 